Uwe Fleckner, Maike Steinkamp, Hendrick Ziegler, Der Sturm der Bilder. Zerstörte und zerstörende Kunst von der Antike bis in die Gegenwart

Berlin : Akademie Verlag, 2011, 256 p., 69,80€

\title{
Marion Deschamp
}

\section{OpenEdition}

\section{Journals}

Édition électronique

URL : http://journals.openedition.org/ifha/7660

DOI : $10.4000 /$ ifha. 7660

ISSN : 2198-8943

Éditeur

IFRA - Institut franco-allemand (sciences historiques et sociales)

Référence électronique

Marion Deschamp, « Uwe Fleckner, Maike Steinkamp, Hendrick Ziegler, Der Sturm der Bilder. Zerstörte und zerstörende Kunst von der Antike bis in die Gegenwart », Revue de l'IFHA [En ligne], Date de recension, mis en ligne le 15 décembre 2013, consulté le 22 septembre 2020. URL : http:// journals.openedition.org/ifha/7660; DOI : https://doi.org/10.4000/ifha.7660

Ce document a été généré automatiquement le 22 septembre 2020

(CIFHA 


\title{
Uwe Fleckner, Maike Steinkamp, Hendrick Ziegler, Der Sturm der Bilder. Zerstörte und zerstörende Kunst von der Antike bis in die Gegenwart
}

\author{
Berlin : Akademie Verlag, 2011, 256 p., 69,80€ \\ Marion Deschamp
}

Ce recueil collectif est le premier volume de la collection Mnémosyne, dont la vocation est de publier les travaux de l'Internationales Warburg-Kolleg. Celui-ci organise depuis 2005 au Warburg-Haus de Hambourg des rencontres bisannuelles sur diverses thématiques ayant trait au statut de l'art et de l'image. Piloté par le Kunstgeschichtliches Seminar de l'université de Hambourg, le projet rassemble des doctorants et jeunes chercheurs venus de divers horizons disciplinaires (histoire, histoire de l'art, philosophie) et nationaux (européens et extra-européens) pour discuter d'un sujet commun, placé sous la figure tutélaire d'Aby Warburg (1866-1929). Le thème de l'année 2007, qui fait l'objet de ce volume, interrogeait la notion de destruction dans l'art, de l'Antiquité à nos jours, dans quatre espaces principaux : France, Italie, Allemagne et Russie. Les articles rassemblés sont rédigés en anglais ou allemand.

Le titre choisi par les éditeurs, jouant sur un effet de bascule sémantique (Bildersturm/ Sturm der Bilder), dilue plus qu'il ne détermine les frontières notionnelles de la réflexion. Loin de se cantonner au seul phénomène de l'iconoclasme - compris comme une dégradation matérielle de l'objet d'art visant à briser sa prétention à être, être vu et faire sens -, le volume tente en effet d'englober toutes les potentialités de la violence destructrice propre au régime de l'image. Or cette action destructrice peut aussi bien œuvrer contre l'art qu'opérer par les moyens symboliques inhérents à ce dernier (zerstörte und zerstörende Kunst). D'autre part, ce qui est brisé ou ce qu'on cherche à liquider peut être une réalité objectale (physique) ou symbolique (soit, en quelque sorte, métaphysique), appartenir à l'ordre des choses ou des idées, de sorte que l'altération métaphorique du sens d'une œuvre peut avoir autant de potentiel 
destructeur que l'agression ou la suppression physique du corps de l'image, et sa disparition matérielle.

Malgré cette dispersion tout azimut de la matière envisagée, le recueil propose une ligne de force centripète qui fédère les sujets abordés autour de la notion de «destruction productive » (produktive Zerstörung), véritable clef de voûte interprétative des (sinon très) différents articles de l'ouvrage. Et les éditeurs de s'expliquer succinctement sur ce choix dans une introduction commune (p. 1-11). Introduit en théorie de l'art par Horst Bredekamp, le concept est fortement inspiré par la théorie de l'économiste Joseph Schumpeter (1883-1950), qui caractérisait le processus, propre selon lui au capitalisme, de disparition de certaines branches d'activités conjointe à l'apparition de nouveaux secteurs d'activités économiques, par le terme de " destruction créatrice » (schöpferische Zerstörung). Dans son maître-livre consacré à la construction de la basilique Saint-Pierre de Rome et paru en 2000, H. B. qualifiait de perpétuel recommencement les travaux qui, de 1450 à 1650 environs, donnèrent forme au Mons vaticanus, après une consécution d'édifications et démolitions successives. Or, H.B. définissait le résultat de ce processus de construction/destruction/reconstruction non comme la somme additionnelle des travaux réalisés, mais bien plutôt comme la résolution d'une tension paradoxale, obligeant les architectes en quête de reconnaissance à effacer la trace de leurs prédécesseurs, non seulement par élimination du déjà-bâti mais encore subversion ou surimposition des éléments du complexe architectonique.

Cependant, et aussi brillante soit elle, cette compréhension spéculative de l'acte destructeur comme forme démiurgique paradoxale fait problème dès lors que l'on tente de l'élever au rang de paradigme artistique à valeur générale. Or, ceci est précisément l'impression que donnent les éditeurs de l'ouvrage, qui ont peut être cherché par un tour de force à subsumer sous ce concept générique des contributions très variées par leurs sujets et qualités.

La première contribution, due à Uwe Fleckner, s'attèle au processus de démantèlement dont furent l'objet les statues des dirigeants soviétiques dans la ex-RDA, après la chute du Mur. Traçant un parallèle entre ce double effacement matériel et mémoriel de l'héritage communiste et le martèlement des effigies romaines caractérisant la damnatio memoriae des mauvais empereurs, l'auteur analyse la puissance symbolique de liquidation du passé passant par l'agression du corps en effigie (Angriff auf den Bildkörper). Dario Gambori, quant à lui, se saisit de l'effondrement des tours du World Trade Center à New York pour analyser le potentiel symbolique de la destruction de monuments architecturaux dans les conflits politiques actuels. L'auteur attribue à certains monuments une puissance sémaphorique, c'est-à-dire une capacité à porter et exporter du sens. La destruction ciblée des symboles matériels d'un pays servirait, selon lui, d'alternative à l'annihilation guerrière en contexte d'asymétrie, permettant au camp militaire le plus faible d'atteindre par une autre manière le cœur idéologique de l'ennemi. Si cette thèse d'un « équilibre des pouvoirs " par le biais de la destruction symbolique semble relativement convaincante, on se demande par contre quelle est la nature du lien que l'auteur tisse avec les œuvres artistiques représentant la destruction de bâtiments de prestiges ou mettant en scène de manière virtuelle leur démolition. Il nous semble que ces diverses actes de suppression participent de différents régime ontologiques des artefacts humains qu'il serait bon de distinguer. 
Les autres articles du recueil se montrent moins ambitieux théoriquement, mais certains examinent de façon intéressante les modalités polymorphes de l'acte destructeur en art. Susann Holz, notamment, décrit le destin des œuvres d'art emportées comme butin au temps de la Rome impériale et la manière dont ils intégraient le décorum public, avant qu'ils ne soient, quelque fois, restitués à leur propriétaire originel. Godehard Janzing suit, pendant la Révolution française, le sentiment éprouvé par certains d'une perte irrémédiable du patrimoine artistique dû $\mathrm{au}$ « vandalisme artistique ». A partir du néologisme de l'abbé Grégoire (qui «créait le mot pour tuer la chose ») se développe, en effet, la figure du « Vandaliste, destructeur des productions des arts ", qui sert, au delà de la désapprobation de la destruction artistique, de figure politique contestataire.

L'article de Jesper Rasmussen, un peu isolé dans l'ouvrage, essaie (de manière un peu sinueuse) de détailler la politique de destruction ou conservation des objets d'art et les critères de sélection du détruit ou du conservé, dans les écrits de Quatremère de Quincy.

Plusieurs contributions s'efforcent avec plus ou moins de succès, sur des objets variés dans le temps et l'espace, à décortiquer les processus de réemplois, réaménagements reconversions, mais aussi de détournements ou subversions symbolique, observables lorsque un (ou plusieurs artefacts) est extrait de son milieu de signification originel pour servir d'autres fonctions et d'autres sens. Il s'agit donc d'étudier les contours d'une "destruction idéelle» (idéelle Zerstörung, p. 137)) ou d'un iconoclasme des significations (iconoclasm as destruction of meaning, p. 101), qui précède à une recréation $\mathrm{du}$ sens dans un autre contexte. Clemena Antonova examine ainsi la relocalisation des saintes icones russe dans l'espace bolchévique, qui opère (ou cherche à opérer) un désenchantement de l'image religieuse et transforme l'objet de culte en objet esthétique. Erik Wegerhoff effeuille les différents habillements architectoniques (architektonische Bekleidung) du Colisée à Rome pour découvrir les différentes identités sociales et politiques que l'amphithéâtre flavien recouvrit au cours du temps. Andreas Waschbüsch étudie les différentes phases de destruction/reconstruction de la cathédrale de Magdebourg durant le Moyen Age, et Anke Blümm les modifications structurelles et décoratives que subit la maison d'Arnold Zweig, architecte juif avantgardiste, lorsqu'elle passa à la main d'un dirigeant du IIIe Reich.

Les trois derniers articles scrutent le potentiel destructeur des Credo artistiques des avant-gardes du XXe siècle, comme réponse à l'expérience traumatique de la guerre : Hanno Ehrlicher choisit les futuristes et Maria Stavrinaki Dada et ses disciples allemands. Enfin, Anja Kregeloh compare l'œuvre de trois artistes (Adolf Luther, Jesse Magee, Pipilotti Rist) utilisant le matériau du verre pour mettre en scène la destruction de la transparence du monde contemporain. Se profile aussi ici la self-prophecy de l'anéantissement de l'art par l'art, posé comme principe génératif de l'activité créatrice postmoderne. 
INDEX

Index chronologique : Epochenübergreifend

Thèmes : Kunstgeschichte

\section{AUTEUR}

\section{MARION DESCHAMP}

Université Lumière Lyon 2 